\title{
Verbreitung und Ätiologie von kraniomandibulären Dysfunktionen (CMD) im Kindes- und Jugendalter
}

\section{Prevalence and Etiology of Temporomandibular Disorders (TMD) In Childhood and Adolescence}

Autor

Christian Hirsch

Institut

Poliklinik für Kinderzahnheilkunde und Primärprophylaxe, Universität Leipzig

\section{Schlüsselwörter}

kraniomandibuläre Dysfunktionen, Kiefergelenk

Key words

craniomandibular dysfunction, temporomandibular joint

\author{
Bibliografie \\ DOI https://doi.org/10.1055/a-0830-1484 \\ Inf Orthod Kieferorthop 2019; 51: 40-49 \\ (c) Georg Thieme Verlag KG Stuttgart · New York \\ ISSN 0020-0336
}

Korrespondenzadresse

Prof. Dr. Christian Hirsch, M.Sc. (Epidemiologie)

Poliklinik für Kinderzahnheilkunde und Primärprophylaxe

Universitätszahnmedizin Universität Leipzig

Liebigstraße 12, Haus I

04103 Leipzig

Tel.: +49/341/9721 070, Fax: + 49/341/9721079

christian.hirsch@medizin.uni-leipzig.de

http://kinderzahnheilkunde.uniklinikum-leipzig.de/

\section{ZUSAMMENFASSUNG}

Kinder und Jugendliche sind in der zahnärztlichen Sprechstunde als CMD-Patienten kaum anzutreffen, obwohl die Symptome bereits in diesem Alter weitverbreitet sind. Das Erstauftreten von CMD besonders bei Mädchen während der Pubertät sowie die höhere Prävalenz bei Frauen während des Reproduktionsalters lassen die hormonellen Umstellungen während der Pubertät als ätiopathogenetisch bedeutsam erscheinen. Die Mechanismen sind noch nicht klar, es scheint aber ein Mangel an frei verfügbaren Sexualhormonen während der Pubertät ausschlaggebend zu sein. Hierbei könnte es sich um ein grundlegendes Muster für viele unklare Schmerzsymptome handeln, da damit klinische Beobachtungen erklärt werden können: der typische Alters- und Geschlechtsverlauf von CMD, die psychosoziale Charakteristik von CMD-Schmerzpatienten sowie die hohe Koinzidenz der verschiedenen Schmerzarten. Dagegen spielen morphologische Faktoren und deren Veränderungen, z. B. infolge kieferorthopädischer Behandlungen, in der Ätiologie von CMD während des Jugendalters kaum eine Rolle. Darüber hinaus gibt es Erkenntnisse über genetische Ursachen der CMD, ein Bereich, der ebenfalls Gegenstand aktueller Forschungen ist.

\section{ABSTRACT}

Children and adolescents are rarely to be found as CMD patients in the dentist's practice, even though the symptoms are already widespread at this age. The first appearance of CMD especially in girls during puberty as well as the higher prevalence in women during the reproductive age, indicate that the hormonal changes during puberty appear to be etiopathogenetically significant.

The mechanisms are not yet clear, but a lack of freely available sex hormones during puberty seems to be crucial. This may be a basic pattern for many unclear pain symptoms, as it can explain clinical observations: the typical age and sex history of CMD, the psychosocial characteristics of CMD pain patients, and the high coincidence of different types of pain. In contrast, morphological factors and their changes, e. g. as a result of orthodontic treatment, play hardly any role in the etiology of CMD during adolescence. In addition, there is evidence about the genetic causes of CMD, an area that is also the subject of current research.

\section{Einleitung}

Die Tatsache, dass Kinder und Jugendliche eher selten CMD-Patienten in der zahnärztlichen Sprechstunde sind, könnte zu der Annahme führen, dass die muskuloskelettalen Störungen im Kausystem in dieser Altersgruppe weniger verbreitet sind als im Erwach- senenalter. In Deutschland war eine Standortbestimmung zum Ausmaß dieser Erkrankungen, deren Ursachen noch immer weitgehend unklar sind, zunächst auch nur für Erwachsene und Senioren möglich. 
Im Rahmen der bevölkerungsrepräsentativen Dritten Deutschen Mundgesundheitsstudie des Instituts der Deutschen Zahnärzte (DMS III) wurden für rund $10 \%$ (Monatsprävalenz) der Erwachsenen CMD-Schmerzen (also Schmerzen im Bereich der Kiefergelenke, der Kaumuskulatur und angrenzender Strukturen) ausgewiesen, etwa $4 \%$ der Erwachsenen in Deutschland suchten subjektiv eine Behandlung für CMD; bei Senioren waren sowohl Schmerzprävalenz als auch Behandlungsbedarf niedriger als bei Erwachsenen [1].

Etwa jeder 6. Erwachsene wird im Lauf seines Lebens zum CMD-Patienten [2]. Beim Blick in die Literatur zeigt sich allerdings, dass im Jugendalter die Symptomatik weitverbreitet ist (Übersicht in [3]) und dass in diesem Lebensabschnitt zudem wichtige ätiopathogenetische Weichenstellungen aufgrund der gleichzeitigen Wirkung vieler möglicher Einflussfaktoren erfolgen. Der entscheidende Aspekt dabei ist, dass die meisten Erwachsenen, die unter chronischen Schmerzen an den verschiedenen Körperregionen leiden, den Beginn dieser Beschwerden im Jugendalter sehen [4]. Auch bei CMD-Schmerzen werden Risikofaktoren, die deren Auslösung initiieren, im Jugendalter vermutet. Damit wird eines klar: Sollen die Prozesse verstanden werden, die zur Entstehung von CMD-Schmerzen führen, sind Untersuchungen im Jugendalter zwingend erforderlich.

Im 1. Teil dieses Beitrags werden Daten zur Verbreitung, zum Altersverlauf, zu den Beeinträchtigungen sowie zur Behandlungssuche von CMD im Jugendalter dargestellt, im 2. Teil ätiologische Aspekte anhand der aktuellen Literatur beleuchtet.

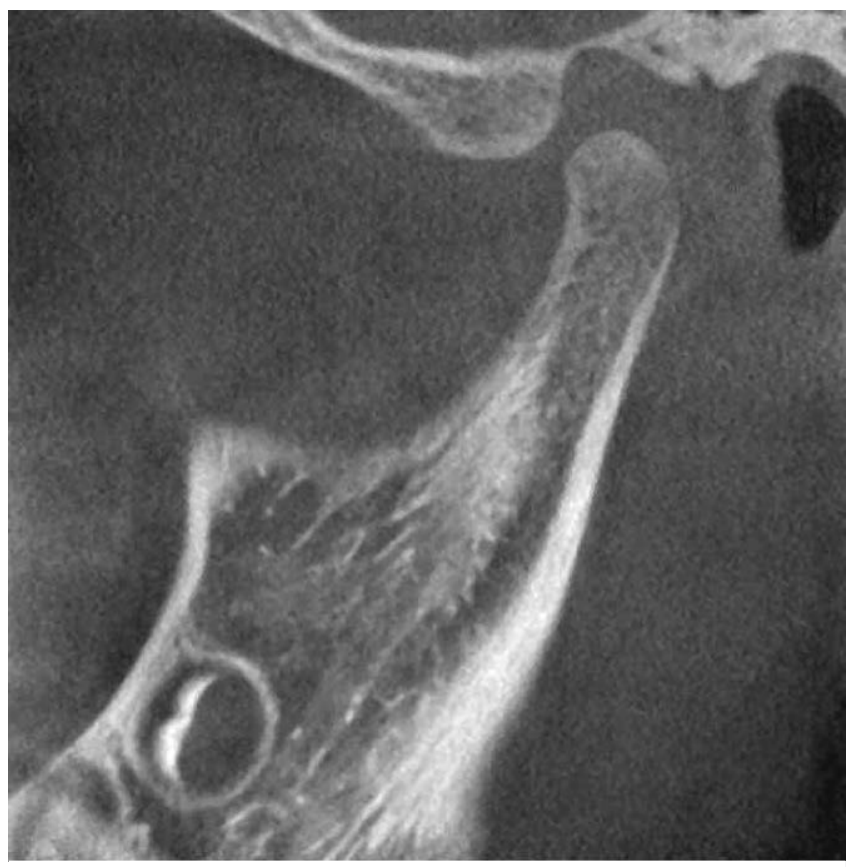

-Abb. 1 Digitales Volumentomogramm des Kiefergelenks einer 15-jährigen Patientin (das Bild zeigt eine normale Konfiguration der knöchernen Gelenkstrukturen). Aus: Hirsch C. Schmerzen im Kausystem. In: Ebinger F, (Hrsg.). Schmerzen bei Kindern und Jugendlichen. Stuttgart: Thieme; 2011: 152-157.

\section{Verbreitung der CMD im Jugendalter}

\section{Diagnostik und Prävalenz}

Die CMD-Diagnostik für das Kindes- und Jugendalter unterscheidet sich nicht grundsätzlich von der bei Erwachsenen. Da die CMD-Diagnosestellung - bis auf die Erfassung von objektivierbaren Gelenkgeräuschen - auf dem Selbstbericht des Patienten basiert, beginnt der Altersbereich für die zuverlässige Erfassung von CMD-Befunden jenseits des 10. Lebensjahrs. Sie setzt sich aus der Anamneseerhebung und der klinischen Untersuchung zusammen, wobei bildgebende Verfahren nur bei speziellen Fragestellungen notwendig sind ( $>$ Abb. 1). Diese Altersgrenze gilt ganz allgemein für selbstberichtete Zustände in den Sozialwissenschaften, z. B. auch in der Lebensqualitätsforschung.

RESEARCH DIAGNOSTIC CRITERIA FOR TEMPOROMANDIBULAR DISORDERS (RDC/TMD)

Es werden 3 Diagnosegruppen unterschieden:

\section{Gruppe I: Schmerzen im Bereich der Kiefermuskulatur}

I. a. myofaszialer Schmerz

I.b. myofaszialer Schmerz mit eingeschränkter Kieferöffnung

\section{Gruppe II: Verlagerungen des Discus articularis}

II.a. Diskusverlagerung mit Reposition bei Kieferöffnung

II.b. Diskusverlagerung ohne Reposition mit eingeschränkter Kieferöffnung

II.c. Diskusverlagerung ohne Reposition ohne eingeschränkte Kieferöffnung

\section{Gruppe III: Gelenkerkrankungen}

III.a. Arthralgie

III.b. Arthritis des Kiefergelenks

III.c. Arthrose des Kiefergelenks

Für das sehr heterogene Krankheitsbild CMD haben sich als diagnostischer Standard die Research Diagnostic Criteria for Temporomandibular Disorders (RDC/TMD) national wie international auch im Jugendalter etabliert [5]. Viele Studien nutzen zudem die Einteilung der CMD-Befunde gemäß dem in Europa weitverbreiteten Helkimo-Index.

Für CMD im Kindes- und Jugendalter gibt es repräsentative Daten aus Deutschland, die im Rahmen einer bevölkerungsrepräsentativen Studie im Stadtgebiet von Halle/Saale an über 1000 Probanden gewonnen wurden [6]. Danach hatte etwa jeder 7. Jugendliche im Alter von 10-18 Jahren anamnestische CMD-Schmerzen im Zeitraum des letzten Monats ( $>$ Tab. 1), Mädchen bzw. weibliche Jugendliche waren davon tendenziell häufiger betroffen. Im Altersverlauf (10/11-16/18 Jahre) nahmen anamnestische CMD-Schmerzen insbesondere bei weiblichen Probanden signifikant zu, bei Jungen tendenziell ab ( $\triangleright$ Abb. 2).

Aufgrund dieser Beschwerden haben 2,1\% der Probanden einen Arzt oder Zahnarzt aufgesucht (subjektiver Behandlungsbedarf). Akute Ohrprobleme (als differenzialdiagnostische Ursache für Schmerzen im Ohrbereich) wurden von weniger als $1 \%$ der Probanden angegeben. Rund $3 \%$ konnten während der klinischen Untersuchung ihren Mund nicht weiter als 35 mm öffnen. Bei 14\% der 
Probanden fanden sich ein oder mehrere schmerzhafte Areale in der Kaumuskulatur bei Palpation bzw. bei Bewegungen des Unterkiefers, positive Muskelbefunde wurden signifikant häufiger von weiblichen Probanden angegeben ( $\triangleright$ Tab. 1).

\section{HELKIMO-INDEX}

\section{Anamnestischer Dysfunktionsindex}

Man unterscheidet 3 Gruppen:

\section{A0: keine anamnestischen Dysfunktionen:}

- nicht $\mathrm{A} 1$ oder $\mathrm{A} 2$

\section{A1: milde anamnestische Dysfunktion:}

positive Antwort auf die Frage (einzeln oder Kombination) nach:

- Kiefergelenkgeräuschen (Knacken, Reiben)

- Gefühl der Müdigkeit im Kieferbereich bzw. ein Gefühl der Steifheit beim Bewegen des Kiefers oder beim morgendlichen Erwachen

- nicht $\mathrm{A} 2$

\section{A2: schwere anamnestische Dysfunktion:}

positive Antwort auf die Frage (einzeln oder Kombination) nach:

- Schwierigkeiten beim Schließen des Mundes

- Blockaden des Unterkiefers oder Schwierigkeiten beim vollständigen Öffnen des Mundes

- Schmerzen beim Bewegen des Unterkiefers

- Schmerzen im Bereich des Kiefergelenks und/oder der Kaumuskulatur

- Schmerzen im Gesicht, Kiefer, in den Schläfen, vor dem oder im Ohr

- kann auch A1 sein

\section{Klinischer Dysfunktionsindex}

Dieser setzt sich aus 4 Kriterien zusammen, die über ein Punktesystem den Dysfunktionsklassen (D0-D3) *

zugeordnet werden:

\section{a. Unterkieferbeweglichkeit:}

- eingeschränkte Mundöffnung

- eingeschränkte Lateralbewegung

- eingeschränkte Protrusion

\section{b. Gelenkfunktion:}

- Kiefergelenkgeräusche (Knacken, Reiben)

- Deviation bei Öffnungsbewegung

\section{c. Palpation der Kaumuskulatur:}

- Anzahl von Stellen in der Kaumuskulatur mit Palpationsempfindlichkeit

\section{d. Palpation des Kiefergelenks:}

- Schmerz bei Palpation von lateral

- Schmerz bei Palpation von dorsal

* www.med-college.de/de/downloads/Helkimo-Index_en.pdf

\section{DATEN ZUR CMD-PRÄVALENZ IM JUGENDALTER}

In Populationsstudien beträgt die Prävalenz von anamnestischen CMD-Schmerzen im Zeitraum des vergangenen Monats über $10 \%$. Schmerzen bei Unterkieferbewegungen oder Palpation in der Kaumuskulatur sind zwischen 1 und $9 \%$ prävalent, in den Kiefergelenken zwischen 1 und $5 \%$. Ein Behandlungswunsch für CMD-Schmerzen besteht bei $2 \%$ der Kinder und Jugendlichen. Anamnestische und klinische CMD-Schmerzen werden häufiger von weiblichen Probanden angegeben. Nicht schmerzhafte Kiefergelenkgeräusche gaben $13 \%$ der Probanden an.

Bei der klinischen Untersuchung wiesen über 20\% der Probanden ein reproduzierbares Knacken auf, Limitationen der Kieferöffnung wurden anamnestisch von $4 \%$ der Probanden angegeben und klinisch bei $3 \%$ gemessen ( $<35 \mathrm{~mm}$ ).

Diese Prävalenzdaten zeigen eindeutig, dass CMD-Symptome im Jugendalter ebenso verbreitet sind wie bei Erwachsenen.

Schmerzen in den Kiefergelenken bei Palpation der Gelenkstrukturen bzw. beim Bewegen des Unterkiefers waren etwa bei jedem 12. Probanden anzutreffen, hier wurden keine Geschlechtsunterschiede festgestellt ( $\triangleright$ Tab. 1). Zudem waren viele sowohl der schmerzhaften als auch der nicht schmerzhaften CMD-Symptome rechts- und linksseitig miteinander korreliert, die Schmerzen im Gesichtsbereich auch mit Beschwerden in anderen Körperregionen. Beides spricht dafür, dass es sich bei CMD eher um ein generalisiertes Problem als um eine lokale Störung handelt.

CMD-Befunde sind damit in der Altersgruppe zwischen 10 und 18 Jahren in Deutschland praktisch ebenso verbreitet wie bei Erwachsenen.

\section{Behandlungsbedarf der CMD im Jugendalter}

Die doch recht hohe Prävalenz muskuloskelettaler Beschwerden im orofazialen Bereich bei den untersuchten Kindern und Jugendlichen (ca. 15\% mit anamnestischen und rund 10\% mit klinischen Schmerzen) mag den Zahnarzt zunächst verwundern, weil dafür durch Angehörige dieser Altersgruppe eher selten eine Behandlung gesucht wird.

Im allgemeinmedizinischen bzw. pädiatrischen Sektor ist dies jedoch nicht ungewöhnlich, hier sind außerhalb von Epidemiezeiten mit Infekten der oberen Luftwege oder Durchfallerkrankungen Schmerzen am Bewegungsapparat - insbesondere an den Gelenken - der dritthäufigste Grund für die Vorstellung eines Kindes bzw. Jugendlichen [7]. In der vorliegenden Studie haben innerhalb eines Monats etwa $2 \%$ der Probanden aktiv eine Behandlung für ihre orofazialen Schmerzen gesucht (subjektiver Behandlungsbedarf).

Hochgerechnet auf die Gesamtbevölkerung in Deutschland nehmen etwa 100000 bis 150000 jugendliche Patienten monatlich eine CMD-Behandlung in Anspruch. 
- Tab. 1 Gewichtete Prävalenz von Schmerzen, Kiefergelenkgeräuschen und Einschränkungen der Mundöffnung (anamnestisch und klinisch, mit Test auf Geschlechtsunterschiede) bei der Halleschen Stichprobe ( $N=1011$, Altersbereich 10-18 Jahre). Aus [6].

\begin{tabular}{|c|c|c|c|c|}
\hline & $\begin{array}{l}\text { gesamt }(\mathrm{N}=1011) \% \\
\text { (N) }[95 \%-\mathrm{KI}]\end{array}$ & $\begin{array}{l}\text { männlich (N=486) \% } \\
\text { (N) [95\%-KI] }\end{array}$ & $\begin{array}{l}\text { weiblich }(\mathrm{N}=525) \% \\
\text { (N) [95\%-KI] }\end{array}$ & $\begin{array}{l}\text { P } \\
\text { (X2-Test) }\end{array}$ \\
\hline \multicolumn{5}{|l|}{ Anamnese } \\
\hline anamnestische CMD-Schmerzen im letzten Monat & $14,9(156)[11,9 ; 18,4]$ & $13,8(61)[10,0 ; 18,6]$ & $15,9(95)[11,5 ; 21,5]$ & 0,49 \\
\hline Kiefergelenkgeräusche im letzten Monat & $12,6(149)[9,4 ; 16,6]$ & $11,3(61)[6,9 ; 17,9]$ & $13,8(88)[11,2 ; 16,8]$ & 0,48 \\
\hline Mundöffnungsbehinderungen im letzten Monat & $3,6(37)[2,2 ; 5,6]$ & $3,4(15)[1,4 ; 8,1]$ & $3,7(22)[2,2 ; 6,2]$ & 0,59 \\
\hline \multicolumn{5}{|l|}{ klinische Untersuchung } \\
\hline Schmerzen in der Kaumuskulatur bei Palpation/Bewegung & $13,9(156)[9,9 ; 19,1]$ & $11,5(60)[6,6 ; 19,3]$ & $16,1(96)[12,0 ; 21,2]$ & 0,01 \\
\hline Schmerzen in den Kiefergelenken bei Palpation/Bewegung & $8,3(86)[5,9 ; 11,5]$ & $8,7(38)[5,0 ; 14,8]$ & $7,9(48)[5,2 ; 11,8]$ & 0,45 \\
\hline reproduzierbares Knacken bei vertikalen/horizontalen Bewegungen & $22,6(248)[15,6 ; 31,7]$ & $19,6(107)[10,8 ; 32,9]$ & $25,5(141)[17,7 ; 35,4]$ & 0,07 \\
\hline Krepitation bei vertikalen/horizontalen Bewegungen & $2,9(34)[1,6 ; 5,4]$ & $2,9(18)[1,3 ; 6,3]$ & $3,0(16)[1,7 ; 5,4]$ & 0,56 \\
\hline Mundöffnungsbehinderungen ( $<35 \mathrm{~mm}$ ) & $3,0(31)[2,2 ; 4,3]$ & $3,3(16)[2,2 ; 5,0]$ & $2,8(15)[1,4 ; 5,4]$ & 0,65 \\
\hline KI: Konfidenzintervall & & & & \\
\hline
\end{tabular}

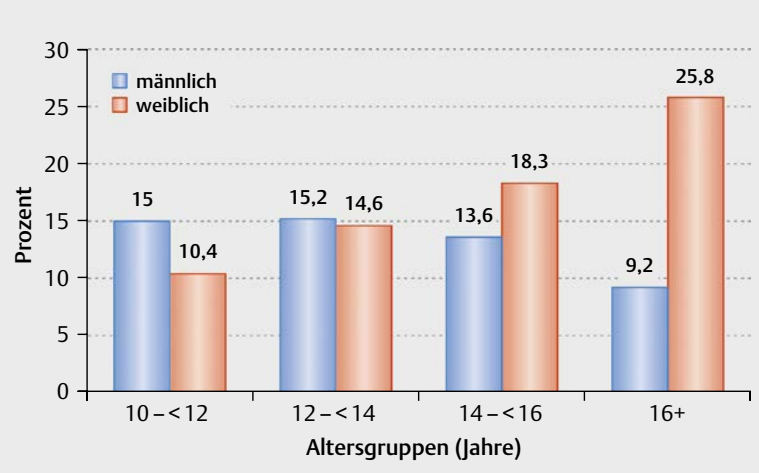

Abb. 2 Altersverlauf von anamnestischen CMD-Schmerzen zwischen 10 und 18 Jahren bei der Halleschen Stichprobe $(\mathrm{N}=1011)$ (Daten aus [6]).

Tendenziell sind Mädchen häufiger betroffen, damit etabliert sich der später im Erwachsenenalter bestehende Geschlechtsunterschied in der Behandlungssuche bezogen auf die Allgemeinbevölkerung bereits im Verlauf des Kindes- und Jugendalters. Insgesamt stimmen diese Angaben zum Behandlungsbedarf gut mit bereits publizierten Angaben sowie Expertenschätzungen überein, wonach etwa 2-5\% der Kinder und Jugendlichen einen Behandlungsbedarf bezüglich einer CMD aufweisen [5].

Unbekannt ist bislang jedoch der Anteil, der zur Vermeidung von Chronifizierungstendenzen tatsächlich im Jugendalter behandelt werden müsste (normativer Behandlungsbedarf). Dass damit auch im Kindes- und Jugendalter gerechnet werden muss, zeigen Studien, wonach mehr als die Hälfte der Kinder mit idiopathischen Muskelschmerzen ein chronisches Schmerzgeschehen entwickeln [8]. Aller Wahrscheinlichkeit nach ist der normative Behandlungsbedarf deutlich größer als der subjektive. Es ist bislang aber unklar, was getan werden müsste, um eine Chronifizierung der Beschwerden zu vermeiden.
Wichtige Anhaltspunkte für die Einschätzung der Behandlungsnotwendigkeit ergeben sich aus dem Grad der Beeinträchtigungen infolge orofazialer Schmerzen, da funktionelle Einschränkungen im Prozess der Chronifizierung offenbar eine größere Rolle spielen als die momentane Schmerzintensität [9]. In der anamnestischen Befragung fühlte sich immerhin $1 / 4$ der jugendlichen Probanden durch orofaziale Schmerzen „stark“ oder „sehr stark“ beeinträchtigt. In der Einschätzung der Jaw Disability[10], also der durch die Beschwerden verursachten Limitationen im Kausystem, waren diese qualitativ (welche Tätigkeiten sind limitiert) im Vergleich mit Erwachsenen sehr ähnlich, quantitativ (wie häufig treten die Limitationen auf) sogar etwas stärker ausgeprägt.

Unklar ist jedoch, warum bei den untersuchten Jugendlichen der Grad der subjektiv angegebenen Beeinträchtigungen nicht mit der Behandlungssuche korreliert. Dies kann damit zusammenhängen, dass sich die CMD als heterogene Erkrankung ganz unterschiedlich auf das psychologische Profil der Betroffenen auswirkt, was auch die wiederholte Inanspruchnahme von CMD-Sprechstunden beeinflusst [11]. Es scheint, Patienten mit muskulären CMD und solche mit bereits chronifizierten Schmerzen haben für die erneute Vorstellung in der Sprechstunde eine erhöhte Wahrscheinlichkeit. Solche Mechanismen beginnen sich im Jugendalter aber erst zu etablieren.

Die Relevanz der CMD wird auch im Jugendalter nicht von den physischen, sondern insbesondere von psychosozialen Faktoren bestimmt.

\section{Verlauf der CMD über die Lebensspanne}

Um ein Gesamtbild der CMD über die Lebensspanne zu zeichnen, wurden die Daten aus Halle/Saale zur Prävalenz der CMD im Jugendalter mit Angaben bei Erwachsenen und Senioren in Deutschland aus der Dritten Deutschen Mundgesundheitsstudie (DMS III) verglichen. Insgesamt liegen hier Daten aus der deutschen Bevölke- 
rung von über 3000 repräsentativ ausgewählten Probanden über einen Altersbereich von 10-75 Jahren vor, die mit einheitlicher Methodik untersucht worden sind.

Da es in der Literatur bislang keine ähnliche Datenerhebung zur CMD-Prävalenz über einen so weiten Altersbereich mit Aufschlüsselung der einzelnen Altersgruppen gibt, sind unmittelbare Vergleiche mit der Literatur nicht möglich. Dargestellt wird hier die CMD-Prävalenz nach dem Helkimo-Index für schwere anamnestische Dysfunktionen (A2), weil hierunter jeweils die klinisch bedeutsamen Schmerzbefunde subsumiert sind.

Es zeigt sich folgendes Bild: Während im Kindesalter (gemeint ist hier präpubertär) die anamnestische CMD-Prävalenz bei beiden Geschlechtern etwa gleich groß ist (Jungen sind etwas, jedoch nicht signifikant häufiger vertreten), weist das weibliche Geschlecht ab dem Eintritt in die Pubertät konstant eine etwa doppelt so hohe Prävalenz dieser Symptome auf. Erst im höheren Lebensalter (bei Senioren) gehen dann die anamnestischen Befunde wieder zurück und die Geschlechtsunterschiede verschwinden ( $\triangleright$ Abb. 3).

Dieser „Prävalenzgipfel“ im Reproduktionsalter lässt sich bei Frauen auch für andere Schmerzzustände wie Migräne beobachten. Letztlich gibt der typische Altersverlauf wichtige Hinweise auf die Ätiologie der Erkrankung, denn die Risikofaktoren, die insbesondere den Geschlechtsunterschied ausmachen, entfalten ihre wesentliche Wirkung im Verlauf des Reproduktionsalters. Es ist äußerst unwahrscheinlich, dass diese Faktoren morphologischer Natur sind. Entscheidend ist letztlich, dass eine Theorie zur Ätiologie von CMD die Alters- und Geschlechtsverteilung ( $>$ Abb. 3) biologisch plausibel erklären können muss.

Eine Theorie zur Ätiologie von CMD muss die Alters- und Geschlechtsverteilung biologisch plausibel erklären können.

\section{CMD - ein Gesundheitsproblem bei Mädchen und Frauen?}

CMD sind sowohl in Bezug auf die Prävalenz als auch die Behandlungssuche hauptsächlich ein Gesundheitsproblem von Frauen. Obwohl die Symptomhäufigkeit in der Bevölkerung in Bezug auf das Geschlechtsverhältnis Frauen zu Männern (\Abb. 3) ca. 2:1 beträgt, sind in klinischen Patientenpopulationen rund 4 von $5 \mathrm{~Pa}$ -

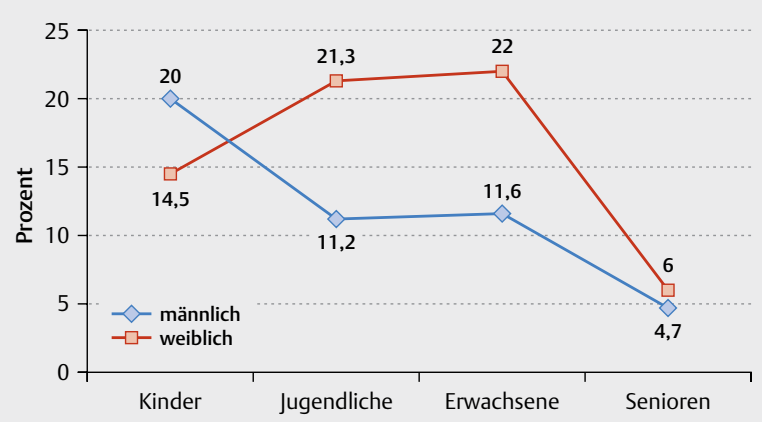

Abb. 3 Prävalenz anamnestischer CMD-Befunde bei Kindern und Jugendlichen (Hallesche Studie), Erwachsenen und Senioren in Deutschland (DMS III) (Daten aus [6]). tienten, die eine CMD-Behandlung in Anspruch nehmen, weiblichen Geschlechts, was jeder Kliniker bestätigen wird.

Es gibt viele Hinweise darauf, dass Frauen unter orofazialen Schmerzen mehr leiden als Männer. Gleiches gilt für Jugendliche; eine Studie aus Schweden bestätigte diese Beobachtungen mit der Schlussfolgerung:

„Girls reporting TMD pain had significantly greater impact on behavioral and psychosocial factors than boys. Almost one third of older girls, compared to one out of 10 older boys, reported school absences and analgesic consumption because of their TMD pain“ [12].

Der Umstand, dass Mädchen vor dem Pubertätsalter nicht, danach aber durchweg häufiger als Jungen CMD-Symptome angeben, spricht für eine tatsächlich erhöhte Prädisposition von CMD beim weiblichen Geschlecht. Aus den Daten aus Schweden und Halle/ Saale kann aber sicher abgeleitet werden, dass sich die typischen Geschlechtsunterschiede hinsichtlich des Schmerzgeschehens durch eine CMD bereits im Jugendalter etablieren, zeitlich also vor der im Erwachsenenalter einsetzenden Doppelbelastung der Frauen durch Beruf und Familie, die als Hypothese für die allgemein höhere Schmerzprävalenz bei Frauen schon lange diskutiert wird. Unklar bleibt weiterhin, ob Mädchen und Frauen wirklich mehr Schmerzen aufweisen oder ob sie nur in größerem Umfang als beim männlichen Geschlecht über die verschiedenen Symptome berichten.

\section{FAZIT}

Es gibt einen Anstieg der CMD-Prävalenz im Jugendalter, der sich im Wesentlichen rekrutiert aus der Zunahme von Schmerzwahrnehmungen bei Mädchen sowie aus der Zunahme von nicht schmerzhaften Gelenkgeräuschen bei Mädchen und Jungen (insofern man diesen einen Krankheitswert zuschreibt). Die relativen Geschlechtsunterschiede hingegen ergeben sich aus der subjektiv empfundenen Zunahme von Schmerzen bei Mädchen sowie aus einer signifikanten Abnahme subjektiv empfundener und tatsächlich festgestellter Schmerzbefunde bei Jungen. Am Ende des Jugendalters sind schließlich als Folge der stattfindenden vielfältigen biologischen und psychosozialen Veränderungen die bei Erwachsenen beobachteten Geschlechtsunterschiede manifest - in Bezug auf die Prävalenz der CMD, die Beeinträchtigungen sowie die Inanspruchnahme einer Behandlung.

\section{Ätiologische Aspekte der CMD im Jugendalter Aktuelle Probleme der CMD-Forschung}

Die weit überwiegende Mehrheit der Studien zu CMD im Jugendalter sind Querschnittserhebungen. Bezüglich der Ätiologie der CMD können daraus keine endgültigen Beweise für Ursachen abgeleitet werden, allein schon wegen des fehlenden Nachweises über die zeitliche Abfolge von Ursache und Wirkung.

Was jedoch immer wieder erfolgt, ist eine Extrapolation von beobachteten Assoziationen auf ein Ursachengefüge. Am Beispiel zum Zusammenhang zwischen orthopädischen Befunden und CMD kann das exemplarisch gezeigt werden. Eine Literaturübersicht hat 

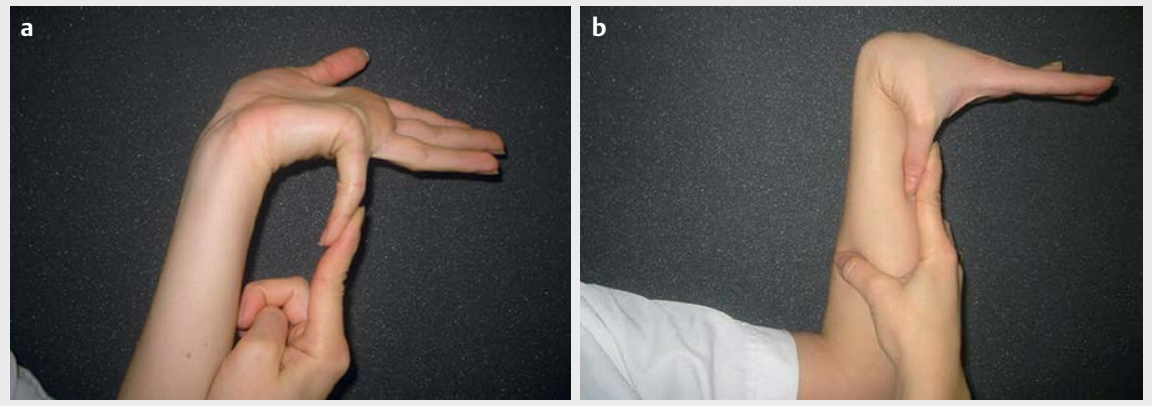

- Abb. 4 Bestimmung der allgemeinen Gelenküberbeweglichkeit (hier an Daumen und kleinem Finger), die als Risikofaktor für Diskusverlagerungen im Kiefergelenk angesehen wird.

- Tab. 2 Longitudinale Studien zur CMD im Kindes- und Jugendalter (Daten aus [6]).

\begin{tabular}{|l|l|l|l|l|l|l|}
\hline $\begin{array}{l}\text { Publika- } \\
\text { tionsjahr }\end{array}$ & N & $\begin{array}{l}\text { Alter der } \\
\text { Probanden (Jahre) }\end{array}$ & $\begin{array}{l}\text { Dauer der } \\
\text { Studie (Jahre) }\end{array}$ & Region & $\begin{array}{l}\text { Einbeziehung der } \\
\text { Pubertätsentwicklung bzw. } \\
\text { hormoneller Faktoren }\end{array}$ \\
\hline 2009 & 1018 & $11-12$ & 20 & South Wales, UK & klinische Untersuchung & nein \\
\hline 2007 & 2255 & $12-19$ & 3 & Schweden & klinische Untersuchung & nein \\
\hline 2007 & 1996 & 11 & 3 & Washington, USA & klinische Untersuchung & ja \\
\hline 2004 & 128 & 15 & 8 & Finnland & Fragebogen & nein \\
\hline 2004 & 97 & 7 & 13 & Finnland & klinische Untersuchung & nein \\
\hline 1999 & 402 & $7-15$ & 3 & Schweden & Fragebogen & nein \\
\hline 1997 & 361 & 13 & 12 & Schweden & klinische Untersuchung & nein \\
\hline 1996 & 170 & $12-13$ & Japan & klinische Untersuchung & nein \\
\hline 1995 & 210 & $7-20$ & 10 & Holland & klinische Untersuchung & nein \\
\hline 1994 & 84 & 15 & 2 & Schweden & klinische Untersuchung & nein \\
\hline 1986 & 285 & $17-19$ & Schweden & klinische Untersuchung & nein \\
\hline
\end{tabular}

hier zu dem Ergebnis geführt, dass in ungefähr der Hälfte der Publikationen die Ursache von orthopädischen Störungen im Kausystem gesehen wird, in der anderen Hälfte der Fälle umgekehrt [13]. Beide Annahmen sind ohne Kenntnis der zeitlichen Abfolge von Ursache und Wirkung unzulässig.

Für wenige ätiologische Faktoren ergibt sich bereits aus Querschnittserhebungen eine klare Ursache-Wirkungs-Beziehung, bspw. aus der allgemeinen Gelenküberbeweglichkeit als Risikofaktor für Diskusverlagerungen im Kiefergelenk [14], die als angeborener Zustand immer vor der Entwicklung der CMD-Symptome steht ( Abb. 4).

Longitudinale Studien zur CMD im Jugendalter sind bislang nur sehr wenige vorhanden. Eine Übersicht von Studien der letzten 25 Jahre zeigt $>$ Tab. 2. Diese Darstellung beinhaltet nur die bis jetzt (Stand: 01.07.2016) publizierten Endauswertungen der jeweiligen Probandenkollektive. So sind z. B. bei einem Probandenkollektiv aus South Wales (UK), über das 1991 erstmals berichtet wurde, und der Publikation der 20-Jahres-Ergebnisse weitere Zwischenergebnisse publiziert worden. Diese wurden hier nicht berücksichtigt, sodass die tatsächliche Anzahl longitudinaler Kohortenstudien zur CMD im Jugend- und jungen Erwachsenenalter niedriger ist, als es eine PubMed-Recherche ergibt. Hier werden bei Eingabe der Stichworte „longitudinal“ [All Fields] AND „TMD“ [All Fields] AND „adolescence“ [MeSH Terms] immerhin 50 Treffer angezeigt (Stand 01.07.2016).

Gegenüber Querschnittsstudien, in die bis zu mehrere Tausend Probanden einbezogen werden, weisen longitudinale Studien grundsätzlich niedrigere Probandenzahlen auf. Oft handelte es sich um Nachuntersuchungen kieferorthopädischer Patienten [15] oder (erwachsener) Studenten [16], die zwar gut rekrutierbar und dokumentiert sind (mit Behandlungsplänen, Modellen, Röntgenaufnahmen usw.), aber aufgrund ihrer Nichtrepräsentativität für die Allgemeinbevölkerung (sog. fehlende externe Validität) zur ätiologischen CMD-Forschung nur bedingt geeignet sind. So sind z. B. Mädchen und Frauen bei kieferorthopädischen Behandlungen in den meisten Industriestaaten bekanntermaßen deutlich überrepräsentiert. 
Die bisher am längsten nachbeobachteten Kohorten stammen aus Großbritannien bzw. Schweden (jeweils 20 Jahre, vgl. \ Tab. 2). Wesentliche Ergebnisse der Längsschnittuntersuchungen sind:

- CMD-Symptome nehmen über den Zeitverlauf zu, dabei kommt es jedoch zu Fluktuationen.

- Das weibliche Geschlecht ist häufiger betroffen.

- Die bis dato vermuteten morphologischen Risikofaktoren (einschließlich der die Morphologie verändernden kieferorthopädischen Behandlung) haben offenbar keinen Einfluss auf die CMD.

Diese Erkenntnisse nahmen LeResche et al. [17] auf und haben zu Beginn der 1990er-Jahre im Großraum Seattle (USA) die bislang umfangreichste longitudinale Studie (Probandenzahl = 1996) zu Risikofaktoren, die zum Erstauftreten von CMD-Schmerzen im Jugendalter führen, durchgeführt. Danach konnten neben dem weiblichen Geschlecht (Odds Ratio [OR] = 2,0; $95 \%$-Konfidenzintervall $[\mathrm{KI}]=1,2-3,3)$ sowie negativer somatischer und psychologischer Symptome inklusive Somatisierung $(\mathrm{OR}=1,8$; $95 \%-\mathrm{KI}=1,1-2,8)$ auch die Prävalenz weiterer Schmerzzustände außerhalb des Zahn, Mund- und Kiefer-Bereichs (ZMK-Bereich) (OR=3,2; $95 \%$-KI = 1,7$6,1)$ sowie die Unzufriedenheit mit dem eigenen Leben $(O R=4,1$; $95 \%-K I=1,9-9,0)$ als wesentliche Risikofaktoren für das Erstauftreten von CMD-Schmerzen identifiziert werden [18].

Dies ist bislang eine der wenigen Studien im zahnmedizinischen Bereich, die eine Assoziation von CMD zur pubertären Entwicklung überhaupt untersuchte, obwohl dieser Aspekt so naheliegend ist. Die Autoren schlussfolgerten, dass die Pubertätsentwicklung ein besserer Prädiktor für Schmerzen war als das nominale Alter der Probanden, und geben damit gleichzeitig einen Hinweis auf die Bedeutung der hormonellen Veränderungen für die CMD in diesem Lebensabschnitt.

Das Fehlen longitudinaler Studien zur CMD im Jugendalter unter Einbeziehung von Aspekten der Pubertätsentwicklung stellt die wesentliche Limitation in der ätiologischen Forschung dar.

\section{Pubertätsentwicklung und Reproduktionshormone}

In dem sich gegenwärtig abzeichnenden Paradigmenwechsel in der CMD-Forschung - weg von einem mechanistisch basierten und hin zu einem medizinisch basierten Krankheitsmodell [19] - verdichten sich die Hinweise, dass hormonellen Einflüssen bei der Entstehung von CMD-Schmerzen eine entscheidende Bedeutung zukommt:

- Bei der Betrachtung von Studien, die das Pubertätsalter im Querschnitt übergreifen, fällt trotz der unterschiedlichen Methodik auf, dass vor dem Eintritt ins Pubertätsalter generell sehr niedrige Prävalenzraten für CMD angegeben werden, danach jedoch wesentlich mehr [20].

- Während bei Kindern kaum Geschlechtsunterschiede bei CMD beobachtet werden, ist ab dem Jugendalter das weibliche Geschlecht in der Prävalenz, den Beeinträchtigungen und in der Behandlungssuche deutlich überrepräsentiert [12].
- Frauen in den postmenopausalen Lebensjahren zeigen eine niedrigere CMD-Prävalenz im Vergleich zu Frauen im Reproduktionsalter [21].

- Die Prävalenz von CMD-Schmerzen ändert sich im Regelzyklus bei Frauen [22].

- Während der Schwangerschaft ist die Schmerzprävalenz aufgrund hormoneller Wirkungen erniedrigt [23].

- Die Einnahme oraler Kontrazeptiva führt zu einem $20 \%$ größeren Risiko für das Auftreten myoarthropathischer Schmerzen [24].

Die hormonellen Veränderungen während der Pubertät haben vielfache Einflüsse auf Verhalten, Hirnentwicklung und Psyche und natürlich auch auf den Körper. Während bei Mädchen und Frauen Reproduktionshormone als Ursache für die höhere Schmerzprävalenz angesehen werden können [25], gibt es Hinweise darauf, dass männliche Geschlechtshormone sogar eine Schutzwirkung gegenüber Schmerzen aufweisen.

In einer aktuellen Übersichtsarbeit kommen Cairns und Gazerani [26] zu der Schlussfolgerung, dass verschiedene Schmerzzustände, u. a. CMD-Schmerzen, Fibromyalgie bzw. bestimmte Arten von Kopfschmerzen, zwar deutliche Geschlechtsunterschiede zeigen, dass jedoch die zugrunde liegende Pathophysiologie unverstanden ist. Der offensichtliche Zusammenhang von Schmerzen mit dem Reproduktionszyklus bei Frauen lässt eine Wirkung der Geschlechtshormone jedoch als sehr wahrscheinlich erscheinen [27].

Allerdings sind die Befunde diesbezüglich nicht einheitlich. So konnten Kröner-Herwig und Vath in einer Erhebung aus Deutschland, in die primär ca. 2000 Familien einbezogen wurden, zeigen, dass Kopfschmerzen bei Mädchen im Jugendalter nur sehr wenig von der Pubertätsentwicklung beeinflusst waren [28], wobei es sich hier um nicht muskuloskelettale Schmerzen und außerdem um eine postalische Befragung handelte, die trotz der hohen Probandenzahl in ihrer Aussage eingeschränkt bleiben muss.

Die Auffassung, wonach die physische Entwicklung Bedeutung für die Ätiologie der CMD besitzt, wird von verschiedenen Autoren geteilt. Eine amerikanische Studie (Telefon-Survey) an über 7000 Kindern und Jugendlichen kam hinsichtlich der Prävalenz anamnestischer CMD-Befunde zu dem Schluss, dass in Untersuchungen zu potenziellen Ursachen einer CMD besonders die Faktoren, die während der physischen Entwicklung wirken, berücksichtigt werden müssen [17].

Die weibliche Dominanz bei der Inanspruchnahme von CMD-Behandlungen ergibt sich nach aktuellem Kenntnisstand offenbar aus einem dynamischen ökologischen Modell chronischer Schmerzen unter Einbeziehung biologischer und psychosozialer Faktoren, wobei der Wirkung hormoneller Faktoren vor dem Hintergrund der psychosozialen Veränderungen während des Pubertätsalters eine entscheidende Rolle zukommt [25].

Erste Analysen von Daten aus einem von der DFG geförderten Projekt (HEIDY: Hormonelle Einflüsse auf die Inzidenz kraniomandibulärer Dysfunktionen im Jugendalter) im Rahmen der Kinder- und Jugendlichenkohorte des Leipziger Forschungszentrums für Zivilisationserkrankungen (Life Child) zeigen, dass insbesondere der Mangel an frei verfügbaren Sexualhormonen im Serum mit einer erhöhten Wahrscheinlichkeit für Schmerzen im Kausystem verbunden ist. 
Der Zusammenhang von Schmerzen mit dem Reproduktionszyklus bei Frauen lässt eine Wirkung der Geschlechtshormone auch auf die Entwicklung von CMD-Schmerzen als sehr wahrscheinlich erscheinen.

\section{Morphologische Aspekte des Kausystems}

Eher historisch sind Überlegungen zu morphologischen Ursachen der CMD im Kindes- und Jugendalter zu betrachten. Bedingt durch den Zahnwechsel, der seinen Abschluss mit dem 12.-13. Lebensjahr erreicht, kommt es tatsächlich zu vorübergehenden Störungen in der Okklusion (u. a. Störungen bei Protrusionsbewegung und durch Mediotrusionskontakte), die als Ursachen der CMD in der Diskussion standen.

Tatsächlich scheint das Risiko für die Entwicklung einer CMD in der letzten Phase der Gebissentwicklung temporär erhöht zu sein [29]. Unklar ist allerdings, inwieweit diese temporären Störungen tatsächlich langfristig die Funktion des Kausystems beeinträchtigen. Zahnverlust (und damit Strukturverlust im Kausystem) dürfte bei Kindern und Jugendlichen als Faktor in der Ätiopathogenese von CMD kaum eine Rolle spielen, in Deutschland beträgt die M-Komponente des Kariesindexes bei 12-Jährigen 0,03, d. h., nur ca. jedem 30. Kind fehlt ein bleibender Zahn (Angaben aus der DMS IV). Die ätiologische Bedeutung von Zahnverlusten wie auch die systematische Extraktion von 6-Jahr-Molaren für die Entstehung von CMD im Kindes- und Jugendalter ist nicht bekannt.

\section{Kieferorthopädische Behandlung}

Seit Langem schon wird in der zahnmedizinischen Fachliteratur die Frage diskutiert, ob die CMD durch kieferorthopädische Maßnahmen, die Änderungen in der Gebissmorphologie bewirken, positiv beeinflusst oder im Gegenteil sogar provoziert wird. Weit über 2000 Treffer in PubMed bei Eingabe der Stichworte „orthodontics and temporomandibular disorders" ab den 1950er-Jahren sind Beleg dafür.

Obwohl Übersichtsarbeiten zu dem Schluss kommen, dass die kieferorthopädische Therapie das Risiko für die Entwicklung einer CMD nicht erhöht [30], widerlegen dies neuere Studien. So fanden Godoy et al. [31] ein bis zu 3-fach erhöhtes Risiko für die Entwicklung von CMD-Symptomen während der kieferorthopädischen Behandlung von Jugendlichen.

Darüber hinaus sind viele Zahnärzte bzw. Kieferorthopäden der Auffassung, dass eine ungeeignete kieferorthopädische Behandlung eine CMD hervorrufen kann [32]. Es gibt jedoch zunehmend Evidenz dafür, dass die kieferorthopädische Behandlung weder der Prävention noch der Therapie der CMD dient [33] ( $>$ Abb. 5).

Jedoch gibt es seltenere Anomalien wie den einseitigen Kreuzbiss, bei denen aufgrund der geringen Fallzahlen selbst in großen Populationsstudien keine Aussage möglich ist. Eine durch starke Asymmetrien zwangsweise hervorgerufene Abweichung und damit Störung der Kiefergelenkfunktion ist zumindest biologisch nicht unplausibel.

\section{Funktionelle Aspekte und Parafunktionen}

Die Bedeutung parafunktioneller Aktivitäten des Kausystems wie Bruxismus resultiert daraus, dass diese zum einen nicht kariöse

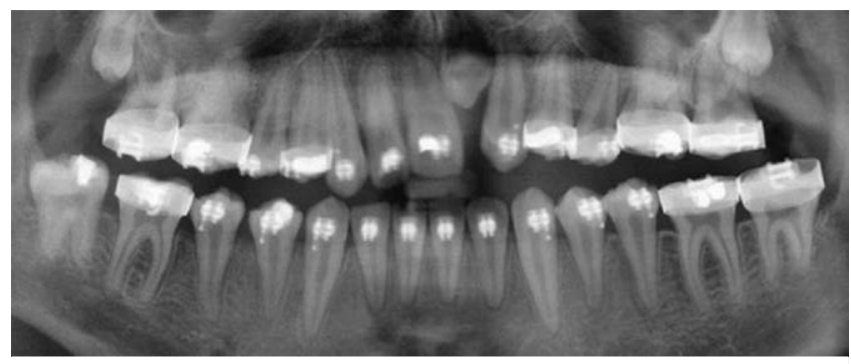

-Abb. 5 Panoramaröntgenaufnahme eines 16-jährigen Patienten (auf den Zähnen sind kieferorthopädische Verankerungselemente aufgeklebt). Aus: Hirsch C. Schmerzen im Kausystem. In: Ebinger F, Hrsg. Schmerzen bei Kindern und Jugendlichen. Stuttgart: Thieme; 2011: 152-157.

Zahnhartsubstanzschäden generieren und zum anderen muskuloskelettale Beschwerden verursachen können [34]. Bei Bruxismus, der manchmal auch als Bestandteil der CMD angesehen wird, handelt es sich um ein vielschichtiges Phänomen mit verschiedenen Ausprägungen. Bruxismus zeigt ähnlich wie CMD eine hohe Verbreitung nicht nur in der erwachsenen Bevölkerung, sondern auch im Kindes- und Jugendalter [33].

Schliff-Facetten an den Zähnen werden bei ca. 20-25\% aller Kinder und Jugendlichen beobachtet [35]. Das gleichzeitige Auftreten von Bruxismus und CMD im Kindes- und Jugendalter ist somit der Tatsache geschuldet, dass beide Phänomene häufig sind. Es spricht nicht zwangsläufig für eine Ursache-Wirkungs-Beziehung, weil auch hierfür longitudinale Studien notwendig sind, die zweifelsfrei zeigen, dass den zuerst beobachteten parafunktionellen Aktivitäten die Schmerzen und Funktionsstörungen folgen.

Die Sprachlautbildung, die im Kindesalter erlernt wird, erfordert ein komplexes Zusammenwirken orofazialer Strukturen. Es gibt Hinweise darauf, dass im Kindesalter bei Störungen der Sprachfunktion auch eine CMD (eingeschränkte Bewegung des Unterkiefers, Gelenkgeräusche, Empfindlichkeit der Kaumuskulatur auf Palpation) gehäuft auftritt [36]. Die Autoren schlussfolgerten daraus auf Wechselwirkungen der verschiedenen orofazialen Funktionen während der Wachstums- und Reifungsphase. Auch hier fehlen longitudinale Daten, um solche Thesen zu belegen, zudem ist die Erfassung von CMD im Kindesalter altersbedingt nicht zuverlässig möglich.

\section{Unfallverletzungen}

Unfälle mit Verletzungen der Kiefer und des Gesichts werden schon lange als wichtiger ätiologischer Faktor für die CMD angesehen, vorrangig für bestimmte CMD-Subtypen wie Myalgie oder Diskusverlagerungen [37], wobei insbesondere Verkehrsunfälle als Ursache einer CMD von Bedeutung zu sein scheinen.

In einer retrospektiven Studie über 1 Jahr an 778 erwachsenen Patienten einer Schmerzklinik wurde neben einem traumatischen Ereignis in der Anamnese auch ein Zusammenhang zwischen orofazialen Schmerzen und chirurgischen Interventionen im orofazialen Bereich festgestellt [38]. Die Autoren schlussfolgerten, dass offenbar beide Ereignisse zu einer Sensibilisierung der Patienten führten. Inwieweit die gerade bei Kindern und Jugendlichen häufig auftretenden dentalen Verletzungen (Zahnfrakturen, Luxationen ohne größere Knochenverletzung) eine ätiologische Bedeutung für die CMD haben, ist aber unklar. 


\section{Genetische Aspekte}

Aus dem Vergleich von objektiven CMD-Prävalenzdaten (Nichtpatienten) verschiedener ethnischer Gruppen lassen sich wichtige Rückschlüsse auf die Ätiopathogenese der CMD ziehen. Eine weitgehende Übereinstimmung in dem Vorkommen objektiver CMD-Befunde in verschiedenen ethnischen Gruppen würde ein von genetischen Faktoren weitgehend unabhängiges Krankheitsmodell mit soziokulturell bedingten Unterschieden bei der Behandlungssuche unterstützen, während differierende physische Befunde eher für ein biologisches (genetisch bedingtes) Krankheitsmodell sprächen.

Ein Vergleich zwischen Deutschland und China zeigte eindeutige Unterschiede in der Prävalenz physischer CMD-Befunde bei Jugendlichen verschiedener ethnischer Gruppen (hier Asiaten und Europäer) auf, die nicht durch äußere Faktoren erklärt werden können [39]. So hatten asiatische Jugendliche mehr CMD-Schmerzen, während die deutschen (kaukasischen) mehr nicht schmerzhafte Befunde wie Diskusverlagerungen aufwiesen. Dies spricht neben den kulturell bedingten Unterschieden beim Selbstbericht von Schmerzen zumindest für eine Beteiligung genetischer Faktoren an der CMD-Ätiopathogenese.

Letztlich führen Überlegungen zum Verstehen eines solch komplexen Phänomens wie Schmerz dazu, die zugrunde liegenden molekulargenetischen Mechanismen zu analysieren. Die Dichte von relevanten Schmerzrezeptoren auf Zell- und Gewebeoberflächen, die Aktivität der Transmitter produzierenden und abbauenden sowie der Reiz wahrnehmenden und übertragenden Strukturen, letztlich die zentrale Verarbeitung der Signale und deren Intensitätsregulation sind weitgehend genetisch determiniert und somit nicht beeinflussbar.

Genetische Analysen scheinen aber neue Perspektiven für das Aufdecken relevanter Assoziationen zu bestimmten Schmerzphänotypen zu eröffnen. Aktuell gibt es dazu ein von der EU gefördertes Projekt, ,... ob nicht kodierende Ribonukleinsäuren (ncRNAs) als Regulatoren der Eiweißsynthese....auf molekulargenetischer Ebene Einfluss auf die individuelle Schmerzverarbeitung haben“ (www.unimainz.de/presse/57854.php). Solche Studien können allerdings nur Teilaspekte der Ätiopathogenese chronischer Schmerzen aufgreifen, vergleichbar mit Puzzleteilen, die erst nach vielen weiteren Projekten zu einem Gesamtbild vervollständigt werden können.

Die Vielzahl der im Jugendalter wirkenden Faktoren verkompliziert die Analyse ätiopathogenetischer Zusammenhänge. Klar ist aber auch: Die Möglichkeit zur Erklärung und Prävention der CMD besteht vorrangig im Jugendalter, wenn diese ursächlichen Mechanismen zu wirken beginnen.

\section{Perspektiven und Resümee}

Der aktuelle Wissensstand zur Ätiologie von CMD muss trotz intensiver Forschung in den letzten Jahrzehnten als unbefriedigend angesehen werden. Viele ätiologische Faktoren im Jugendalter sind noch weitgehend unerforscht, weil die dafür notwendigen aufwendigen longitudinalen Projekte nicht finanziert werden (können). Wenig bekannt sind z. B. die Auswirkungen von physischem und sexuellem Missbrauch im Kindes- und Jugendalter auf das Schmerzgeschehen.

Unter den aktuellen Forschungsprojekten im Erwachsenenalter ist die derzeit laufende OPPERA-Studie ( = Orofacial Pain: Prospective Evaluation and Risk Assessment) hervorzuheben. Das OPPERA-Projekt zielt darauf ab, Ursachen für das Erstauftreten der CMD jenseits des 18. Lebensjahrs sowie die Prozesse der Chronifizierung zu ermitteln. Dabei zeichnet die Studie in Bezug auf die Ursachen der CMD ein sehr heterogenes Bild. Selbst mit den großen Probandenzahlen (2800 Probanden im Alter von 18-44 Jahren werden longitudinal begleitet) ergaben sich bisher wenig deutliche Effekte. Die Autoren schlussfolgerten daher in einem Übersichtsbeitrag:

„... the papers ... demonstrate that CMD is a complex disorder with multiple causes consistent with a biopsychosocial model of illness. It is a misnomer and no longer appropriate to regard CMD solely as a localized orofacial pain condition “ [40].

Diese Erkenntnisse werden aus den Daten im Jugendalter gestützt (HEIDY). Klar ist bislang tatsächlich nur, dass es sich bei CMD nicht um ein lokales, auf das Kausystem reduzierbares Phänomen handelt, sondern um ein allgemeines Störungsbild, was jedoch im Jugendalter seinen Ursprung hat.

Die Unklarheiten bezüglich der Ätiologie der CMD haben letztlich Auswirkungen auf die Therapie. Bedeutsam ist dabei die Unterscheidung zwischen therapeutisch beeinflussbaren und nicht beeinflussbaren Faktoren. Sollte sich herausstellen, dass die in der jugendlichen Entwicklung auftretende Hormonumstellung mit nicht beeinflussbaren Risikofaktoren (z. B. physiologisch bedingte Zunahme des Östrogenspiegels im Blut) - aber eventuell auch mit Schutzfaktoren (z. B. physiologisch bedingte Zunahme des Testosteronspiegels) - einhergeht, so könnte dieses Wissen dazu beitragen, die erreichbaren Behandlungsziele neu zu definieren. Damit könnte ein wichtiger Beitrag dazu geliefert werden, vielfach bestehende (mit dem neuen Wissen dann als überhöht anzusehende) Therapieziele zu korrigieren und die Gefahr einer Überbehandlung zu verringern. Dies würde in erheblichem Maße zur Steigerung der Patientensicherheit beitragen und darüber hinaus helfen, unnötige Ausgaben zu vermeiden, zumal viele der bislang angewandten Behandlungskonzepte der CMD invasiv und kostenintensiv sind. Daher sind im Jugendalter für die CMD ausschließlich reversible und nichtinvasive Therapiemaßnahmen angezeigt (

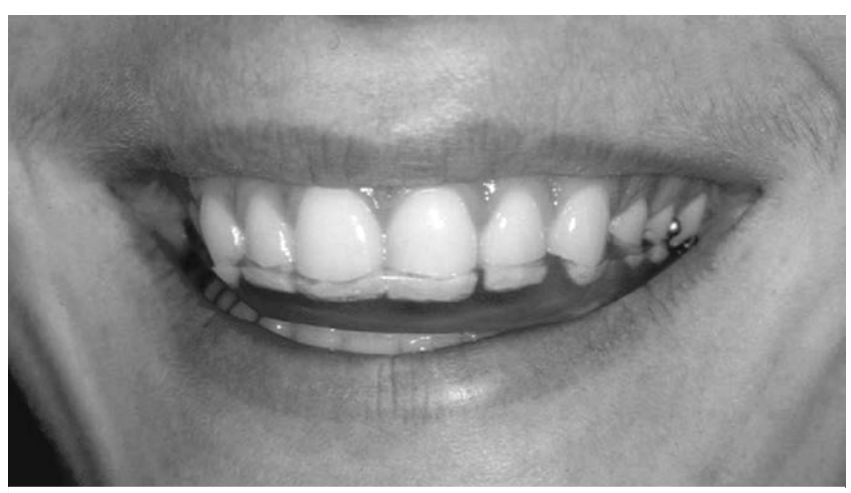

Abb. 6 Schienentherapie bei einer jugendlichen Patientin mit akuten CMD-Schmerzen. Aus: Hirsch C. Schmerzen im Kausystem. In: Ebinger F, (Hrsg.). Schmerzen bei Kindern und Jugendlichen. Stuttgart: Thieme; 2011: 152-157. 
Interessenkonflikt

Die Autoren geben an, dass kein Interessenkonflikt besteht.

\section{Erstveröffentlichung}

Dieser Beitrag wurde erstveröffentlicht in: Zahnmedizin up2date 2016; 6: 579-593.

\section{Literatur}

[1] Micheelis W, Reich E. Dritte Deutsche Mundgesundheitsstudie (DMS III). Köln: Deutscher Ärzte Verlag; 1999

[2] Al-Jundi MA, John MT, Setz JM et al. Meta-analysis of treatment need for temporomandibular disorders in adult nonpatients. J Orofac Pain 2008; 22: 97-107

[3] Hirsch C, Sehrer G. TMD in Children and Adolescents. A Review of the current Literature. In: John M, Hirsch C, Reiber T. (eds.). Epidemiological Research on temporomandibular Disorders. Regensburg: Roderer; 2000

[4] Leboeuf-Yde C, Kyvik KO. At what age does low back pain become a common problem? A study of 29, 424 individuals aged 12-41 years. Spine 1998; 23: 228-234

[5] List T, Wahlund K, Wenneberg B et al. TMD in children and adolescents: prevalence of pain, gender differences, and perceived treatment need. J Orofac Pain 1999; 13: 9-20

[6] Hirsch C. Kraniomandibuläre Dysfunktionen bei Kindern und Jugendlichen [Habilitationsschrift]. Halle: Martin-Luther-Univ. Halle; 2003

[7] Huppertz HI. Gelenkschmerzen im Kindes- und Jugendalter. Monatsschr Kinderheilk 1998; 146: 5-11

[8] Flato B, Aasland A, Vandvik IH et al. Outcome and predictive factors in children with chronic idiopathic musculoskeletal pain. Clin Exp Rheumatol 1997; 15: 569-577

[9] Epping-Jordan JE, Wahlgren DR, Williams RA et al. Transition to chronic pain in men with low back pain: predictive relationships among pain intensity, disability, and depressive symptoms. Health Psychol 1998; 17: 421-427

[10] Dworkin SF, LeResche L. Research diagnostic criteria for temporomandibular disorders: review, criteria, examinations and specifications, critique. J Craniomandib Disord 1992; 6: 301-355

[11] Epker J, Gatchel RJ. Coping profile differences in the biopsychosocial functioning of patients with temporomandibular disorder. Psychosom Med 2000; 62: 69-75

[12] Nilsson IM, Drangsholt M, List T. Impact of temporomandibular disorder pain in adolescents: differences by age and gender. J Orofac Pain 2009; 23: 115-122

[13] Hanke BA, Motschall E, Turp JC. Association between orthopedic and dental findings: what level of evidence is available? J Orofac Orthop 2007; 68: 91-107

[14] Hirsch C, John MT, Stang A. Association between generalized joint hypermobility and signs and diagnoses of temporomandibular disorders. Eur J Oral Sci 2008; 116: 525-530

[15] Dibbets JM, van der Weele LT. Signs and symptoms of temporomandibular disorder (TMD) and craniofacial form. Am J Orthod Dentofacial Orthop 1996; 110: 73-78

[16] Onizawa $\mathrm{K}$, Yoshida $\mathrm{H}$. Longitudinal changes of symptoms of temporomandibular disorders in Japanese young adults. J Orofac Pain 1996; 10: 151-156

[17] LeResche L, Mancl LA, Drangsholt MT et al. Predictors of onset of facial pain and temporomandibular disorders in early adolescence. Pain 2007; 129: 269-278
[18] LeResche L, Mancl LA, Drangsholt MT et al. Relationship of pain and symptoms to pubertal development in adolescents. Pain 2005; 118: 201-209

[19] Greene CS, Laskin DM. Temporomandibular disorders: moving from a dentally based to a medically based model. J Dent Res 2000; 79: 1736-1739

[20] Hirsch C, John M. Prävalenz kraniomandibulärer Dysfunktionen (CMD) bei Kindern und Jugendlichen. Teil 1: Schmerzbefunde. Dtsch Zahnärztl Z 2003; 58: 589-592

[21] Von Korff M, Dworkin SF, Le Resche L et al. An epidemiologic comparison of pain complaints. Pain 1988; 32: 173-183

[22] LeResche L, Mancl L, Sherman J] et al. Changes in temporomandibular pain and other symptoms across the menstrual cycle. Pain 2003; 106: 253-261

[23] LeResche L, Sherman J], Huggins K et al. Musculoskeletal orofacial pain and other signs and symptoms of temporomandibular disorders during pregnancy: a prospective study. J Orofac Pain 2005; 19: 193-201

[24] LeResche L, Saunders K, Von Korff MR et al. Use of exogenous hormones and risk of temporomandibular disorder pain. Pain 1997; 69: 153-160

[25] Fillingim R, Ness T. The Influence of menstrual Cycle and Sex Hormones on Pain Response in Humans. In: Fillingim R. (ed.). Sex, Gender, and Pain. Seattle: IASP; 2000: 191-207

[26] Cairns BE, Gazerani P. Sex-related differences in pain. Maturitas 2009; 63: 292-296

[27] Wang J, Chao Y, Wan Q et al. The possible role of estrogen in the incidence of temporomandibular disorders. Med Hypotheses 2008; 71: 564-567

[28] Kröner-Herwig B, Vath N. Menarche in girls and headache - a longitudinal analysis. Headache 2009; 49: 860-867

[29] Deng YM, Fu MK, Hagg U. Prevalence of temporomandibular joint dysfunction (TMJD) in Chinese children and adolescents. A crosssectional epidemiological study. Eur J Orthod 1995; 17: 305-309

[30] Luther F, Layton S, McDonald F. Orthodontics for treating temporomandibular joint (TMJ) disorders. Cochrane Database Syst Rev 2010; 7 : CD006541

[31] Godoy F, Rosenblatt A, Godoy-Bezerra J. Temporomandibular disorders and associated factors in Brazilian teenagers: a cross-sectional study. Int J Prosthodont 2007; 20: 599-604

[32] Mao Y, Duan XH. Attitude of Chinese orthodontists towards the relationship between orthodontic treatment and temporomandibular disorders. Int Dent J 2001; 51: 277-281

[33] Hirsch C. No increased risk of temporomandibular disorders and bruxism in children and adolescents during orthodontic therapy. J Orofac Orthopedics 2009; 70: 39-50

[34] Glaros AG, Tabacchi KN, Glass EG. Effect of parafunctional clenching on TMD pain. J Orofac Pain 1998; 12: 145-152

[35] Hirsch C, John MT, Lobbezoo F et al. Incisal tooth wear and selfreported TMD pain in children and adolescents. Int J Prosthodont 2004; 17: 205-210

[36] Pahkala R, Laine T, Narhi M et al. Relationship between craniomandibular dysfunction and pattern of speech sound production in a series of first-graders. Eur J Orthod 1991; 13: 378-385

[37] Pullinger AG, Seligman DA. Trauma history in diagnostic groups of temporomandibular disorders. Oral Surg Oral Med Oral Pathol 1991; 71: 529-534

[38] Plesh O, Gansky SA, Curtis DA et al. The relationship between chronic facial pain and a history of trauma and surgery. Oral Surg Oral Med Oral Pathol Oral Radiol Endod 1999; 88: 16-21

[39] Wu N, Hirsch C. Temporomandibular disorders in German and Chinese adolescents. J Orofac Orthop 2010; 71: 187-198

[40] Slade GD, Fillingim RB, Sanders AE et al. Summary of findings from the OPPERA prospective cohort study of incidence of firstonset temporomandibular disorder: implications and future directions. J Pain 2013; 14 (Suppl 12): T116-T124 